\title{
Fardeau Économique Du Paludisme Et Pratiques De Gestion Des Ordures Ménagères : Cas Des Communes De Cocody, Anyama, Port-Bouët, Et Yopougon (Abidjan, Côte d'Ivoire)
}

\author{
Koné-Bodou Possilétya Julie, Doctorante
}

Université Nangui Abrogoua (UNA) / Laboratoire des Sciences de

l'Environnement, Abidjan, Côte d'Ivoire

Ballo Zié, Maitre de Conférences

Université Félix Houphouët Boigny (UFHB)/ UFR Sciences Économique et de Gestion, Abidjan, Côte d'Ivoire

Kouadio Alain Serges, Maitre-Assistant

Université Nangui Abrogoua (UNA) / Laboratoire des Sciences de

l'Environnement, Abidjan, Côte d'Ivoire

Kouamé Kouamé Victor, Maitre-Assistant

Yapi Dopé Armel Cyrile, Maitre-Assistant

Tidou Abiba Sanogo, Professeur Titulaire

Université Jean Lorougnon Guédé / Ecotoxicologie des Milieux Aquatiques, Abidjan, Côte d'Ivoire

\section{Résumé}

Prévenir la santé humaine passe par celle des écosystèmes. La présente étude a pour objectif d'estimer l'influence des modes d'évacuation des ordures ménagères sur le fardeau économique du paludisme. L'évaluation économique selon la fonction dommage a été utilisée sur les données d'enquête transversale de 720 ménages choisies dans quatre communes du District d'Abidjan : Anyama, Cocody, Port- Bouët et Yopougon. L'analyse statistique bi-variée et la régression logistique simple sous le logiciel stata 14 ont permis d'obtenir les résultats. Ceux-ci montrent que le coût du traitement du paludisme est indépendant du mode d'évacuation des eaux usées (Pvalue $=0,997)$. En revanche, il est influencé par les différentes pratiques d'évacuation des ordures solides ( $p$-value $=0,005)$. Le meilleur mode d'évacuation des déchets est celui effectué dans le coffre à ordures. IL fait gagner $38 \%$ sur le coût du traitement du paludisme. L'évacuation par un agent de pré-collecte engendre un bénéfice de $1 \%(\mathrm{P}$-value $=0,039)$ tandis que jeter les ordures dans la rue augmente les charges de traitement du paludisme de 55 
$\%$ (p-value=0,001). La commune péri-urbaine du District, Anyama est la plus concernée par l'évacuation des déchets dans la rue $(52 \%)$. Elle est suivie de la commune résidentielle Cocody $(21 \%)$.

Mots-clés : Fardeau économique, Paludisme, Evacuation des ordures ménagères, Abidjan

\section{Economic Burden of Malaria and Household Waste Management Practices: The Cases of Cocody, Anyama, Port-Bouët, And Yopougon (Abidjan, Côte d'Ivoire)}

\section{Koné-Bodou Possiletya Julie, Doctorante}

Université Nangui Abrogoua (UNA) / Laboratoire des Sciences de

l'Environnement, Abidjan, Côte d'Ivoire

Ballo Zie, Maitre de Conférences

Université Félix Houphouët Boigny (UFHB)/ UFR Sciences Économique et de Gestion, Abidjan, Côte d'Ivoire

Kouadio Alain Serges, Maitre-Assistant

Université Nangui Abrogoua (UNA) / Laboratoire des Sciences de

l'Environnement, Abidjan, Côte d'Ivoire

Kouamé Kouame Victor, Maitre-Assistant

Yapi Dope Armel Cyrile, Maitre-Assistant

Tidou Abiba Sanogo, Professeur Titulaire

Université Jean Lorougnon Guédé / Ecotoxicologie des Milieux Aquatiques, Abidjan, Côte d'Ivoire

Abstract

This paper focuses on estimating the influence of household waste on the economic burden of malaria. Good human healthcare is dependent on ecosystems. Economic evaluation according to the damage function was used on the cross-sectional survey data of 720 households selected in four municipalities of the District of Abidjan: Anyama, Cocody, Port-Bouët, and Yopougon. The bi-varied statistical analysis and the simple logistic regression, using the stata version 14 software, were used to obtain the results of the study. This shows that the cost of malaria treatment is independent of the sewage disposal mode (P-value $=0.997)$. On the other hand, it is influenced by the 
different solid waste disposal practices ( $\mathrm{p}$-value $=0.005)$. When garbage is disposed properly using a garbage bin, it saves $38 \%$ of the cost of treating malaria. Evacuation by a pre-collection officer results in a profit of $1 \%(\mathrm{P}-$ value $=0.039)$, while dumping garbage on the street increases the malaria treatment load by $55 \%$ (p-value $=0.001)$. Disposing waste on the street $(52 \%)$ is very common in Anyama peri-urban municipality. This is followed by the residential municipality Cocody $(21 \%)$.

Keywords: Economic Burden, Malaria, Garbage Disposal, Abidjan

\section{Introduction}

La bonne santé de l'homme passe certainement par celle des écosystèmes (OMS, 2007 ; Charron, 2014). La santé ici se définit comme étant le bien-être économique et social des hommes (Bézy, 2009). Parmi les maladies environnementales connues figure le paludisme. Ensemble avec l'Infection Respiratoire Aigüe (IRA) et la diarrhée, le paludisme représente $60 \%$ des impacts connus de l'environnement sur la santé en Afrique (Opio Odongo, 2013). Au niveau mondial, $42 \%$ des cas du paludisme sont liés à des causes environnementales (OMS, 2007). Cette maladie est aussi considérée par certains auteurs tels que, OMS (2007), Kouadio et al. (2006)et OMS/RBM (2001) comme étant une maladie des pauvres. Selon ces auteurs, le paludisme engendre la pauvreté et est aussi amplifié par cette dernière. Il est prépondérant dans les pays pauvres et est tributaire d'un lourd fardeau économique pour les ménages démunis. Il constitue donc un facteur clé de vulnérabilité pour les populations en situation de précarité (Kouadio et al., 2006).

Pour entrer dans la dynamique des 17 Objectifs de Développement Durable (ODD), programme pour un monde meilleur, pour une prospérité partagée où personne n'est oubliée, il faille s'inscrire dans une prévention du paludisme dans les pays en voie de développement. En effet, éradiquer la faim $(\text { odd } 2)^{1}$, finir avec l'extrême pauvreté (odd1), lutter contre les inégalités(odd10), favoriser l'accès de tous à des énergies renouvelables (odd7), prendre des mésure d'urgences pour lutter contre le changement climatique (odd13), pour ne citer que ceux-ci, suppose de lutter contre toutes les formes d'indigences et d'exclusions où qu'elles soient.

La Côte d'Ivoire fait partie des 192 pays engagés pour le bien-être de tous et des plus vulnérables au travers des ODD. Seulement, elle est en proie au paludisme : $50 \%$ des consultations dans les Etablissements Sanitaires de Premier Contacts (ESPC) et $33 \%$ de tous les décès en milieu hospitalier sont du fait du paludisme (OMS, 2010). Par ailleurs, cette maladie est facteur

${ }^{1}$ Odd2 = Objectif du Développement Durable numéro 2 
d'anémie, d'atteinte neurologique et de complications de la grossesse (OMS, 2010).

L'insalubrité est pour beaucoup dans cette endémie du paludisme en Côte d'Ivoire (Dongo et al., 2006 ; Kouadio et al., 2006 ; Somé et al., 2014). On y observe surtout dans le district d'Abidjan, une cohabitation des ordures ménagères avec la population. Cette cohabitation constatée semble liée aux difficultés de gestion des ordures ménagères. Malgré les efforts financiers, techniques et institutionnels des autorités, le taux de collecte des ordures ménagères est passé de $87 \%$ en 1999 à $44 \%$ en 2011 (Kouamé, 2015). L'évacuation des ordures dans la rue ne semble pas déranger les populations quelques soient les communes. Elles ont l'air d'ignorer les conséquences sanitaires et économiques d'une proximité avec les déchets domestiques (Eviar et al., 2013; Yao -Kouassi \& Gohourou, 2018). Si des études ont pu établir un lien entre certaines modes d'évacuation des ordures ménagères et le paludisme (Brissy et al., 2017), force est de reconnaître qu'elles demeurent encore parcellaires. Plus rares encore sont les analyses économiques faisant allusion à ce lien. En effet, quel est le coût économique supporté par le ménage, quand celui-ci choisi d'évacuer ses ordures, dans un coffre, dans la rue, ou par l'intermédiaire d'un agent pré-collecteur ? Répondre à cette question pourrait amener la population comme les autorités locales à des prises de décisions plus éclairées (Deza, 2017).

La présente étude est une évaluation économique des pratiques de gestion des ordures ménagères dans le district d'Abidjan. Elle a pour objectif principal d'estimer l'influence des modes d'évacuation des ordures ménagères sur le fardeau économique du paludisme des ménages dans les communes de, Anyama, Cocody, Port- Bouët et Yopougon

\section{Objectifs et Hypothèses Objectifs}

Objectif principal : évaluer l'influence des modes d'évacuation des ordures ménagères sur le fardeau économique du paludisme des ménages dans les communes de, Anyama, Cocody, Port-Bouët et Yopougon.

\section{Objectifs spécifiques}

1) Estimer le type de lien existant entre les différents modes d'évacuations des ordures ménagères et la prévalence du paludisme

2) Etablir la fonction dose- réponse entre le paludisme et les modes d'évacuation des ordures ménagères

3) Déterminer les coûts économiques directs du paludisme afférents aux modes d'évacuation des ordures ménagères 


\section{Hypothèses}

1) Le paludisme est positivement corrélé avec le mode d'évacuation des ordures solides.

2) L'évacuation des ordures ménagères dans le coffre expose moins au paludisme et engendre moins de dommages économiques pour le ménage.

\section{Zone et population de l'étude}

Depuis (2001) Abidjan a été érigé en district Autonome par la loi $\mathrm{n}^{\circ}$ 2001-478 du 09 août 2001 portant statut du district d'Abidjan. Le district d'Abidjan est situé sur la façade atlantique au Sud de la Côte d'Ivoire. Sa superficie est de $2119 \mathrm{~km}^{2}$. Il comprend les dix communes de la ville d'Abidjan (Abobo, Adjamé, Attécoubé, Cocody, Marcory, Koumassi, Plateau, Port-Bouët, Treichville, Yopougon) et les sous-préfectures d'Anyama, de Bingerville et de Songon (Figure 1) (N'guettia, 2010). Les communes d'Anyama, Cocody, Yopougon et Port-Bouët ont été choisies pour l'étude. Les critères comme, la position géographique, la connaissance des lieux et le standing de la commune ont influencé le choix de ces communes. Cocody est la commune Résidentielle par excellence du district d'Abidjan tandis qu'Anyama est une commune péri- urbaine. Yopougon et Port- Bouët sont des zones populaires. Yopougon en plus d'être la commune la plus peuplée est située au Nord du district tandis que Port- Bouët se trouve au Sud.

Par ailleurs, ces quatre communes à elles seules regroupent 54, $48 \%$ des résidents des quartiers précaires du District d'Abidjan (PNUD, 2013).

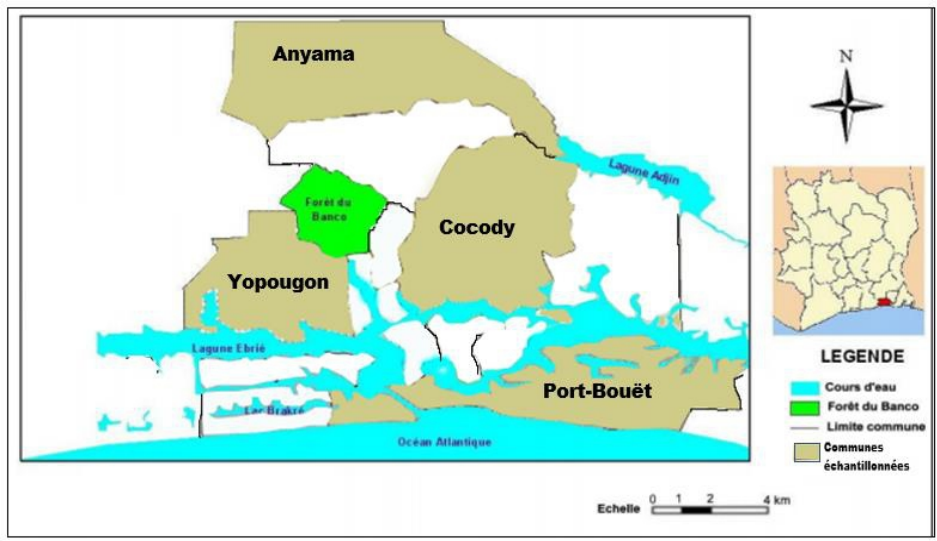

Figure 1. Vue du District d'Abidjan et des quatres communes échantillonées (Anyama, Cocody, Port-bouët Yopougon ) pour l'étude ( Adaptée de N'guettia,2010).

\section{Approche méthodologique Collecte des données}

Une enquête ménage sur 720 ménages a été réalisée pour recueillir les données. La taille de l'échantillon des ménages a été estimée selon la formule suivante : 
Avec :

$\mathrm{N}=\mathrm{A}^{2} \mathrm{P}(1-\mathrm{P}) \mathrm{C} / \mathrm{I}^{2} \quad$ (Vaughan \& Morrow, 1991).

$\mathrm{N}$ : est la taille de l'échantillon,

A : est l'écart type qui est lié au risque $\alpha$, l'erreur de précision de l'estimation, généralement égale à $5 \%$; ce qui donne $\mathrm{A}=1,96$.

$\mathrm{P}$ : est le taux de prévalence maximum attendu pour l'évènement étudié,

$\mathrm{C}$ : représente le coefficient de correction généralement choisi comme égal à 1 ,

I : est le degré de précision de l'échantillonnage ou encore la marge d'erreur d'échantillonnage tolérée.

La prévalence attendue maximum choisie est 30 \% (Koné, 2008). 180 ménages ont été choisis par commune. Puis le choix a tenue compte des quotas des données statistiques du PNUD (2013) sur les quartiers précaires des communes du district d'Abidjan.

Le questionnaire a reposé sur trois rubriques : la morbidité, les caractéristiques sociodémographiques, les dépenses en soins du paludisme et le comportement des ménages face aux ordures ménagères. La femme de ménage a été l'interlocutrice principale et les questions posées concernaient les quinze jours précédant l'enquête. Huit enquêteurs en moyenne par commune essentiellement des doctorants du laboratoire des Sciences de l'Environnement de l'Université Nagui Abrogoua ont été sollicités. Cette enquête s'est déroulée dans les mois de Mai, Juin, Juillet et septembre 2017.

\section{Traitement et analyse des données}

Après la collecte des données à travers la phase de terrain, les fiches d'enquêtes ont été validées, codifiées et les données saisies sur épi-info 7-Le logiciel Epi info est beaucoup utilisé par les professionnels de la santé publique pour la saisie et la gestion des bases de données (Kouadio et al., 2006; Koné, 2008; Center for Disease Control and Prevention (CDC), 2016)-. L'analyse statistique a été effectuée en deux phases. D’abord une analyse statistique bi-variée avec test du Chi2 pour vérifier le lien entre l'avènement du paludisme et les différents modes d'évacuation des ordures ménagères. Ensuite a suivi une régression logistique simple pour estimer les paramètres de cette liaison. L'analyse a été réalisée avec le logiciel Stata version 14, logiciel d'analyses économétriques prisé par les économistes (Ouellet et al., 2005). Le schéma suivant résume toute la méthodologie adoptée par l'étude. La troisième ligne du schéma présente les restitutions de la démarche singulière adoptée (Figure 2). 


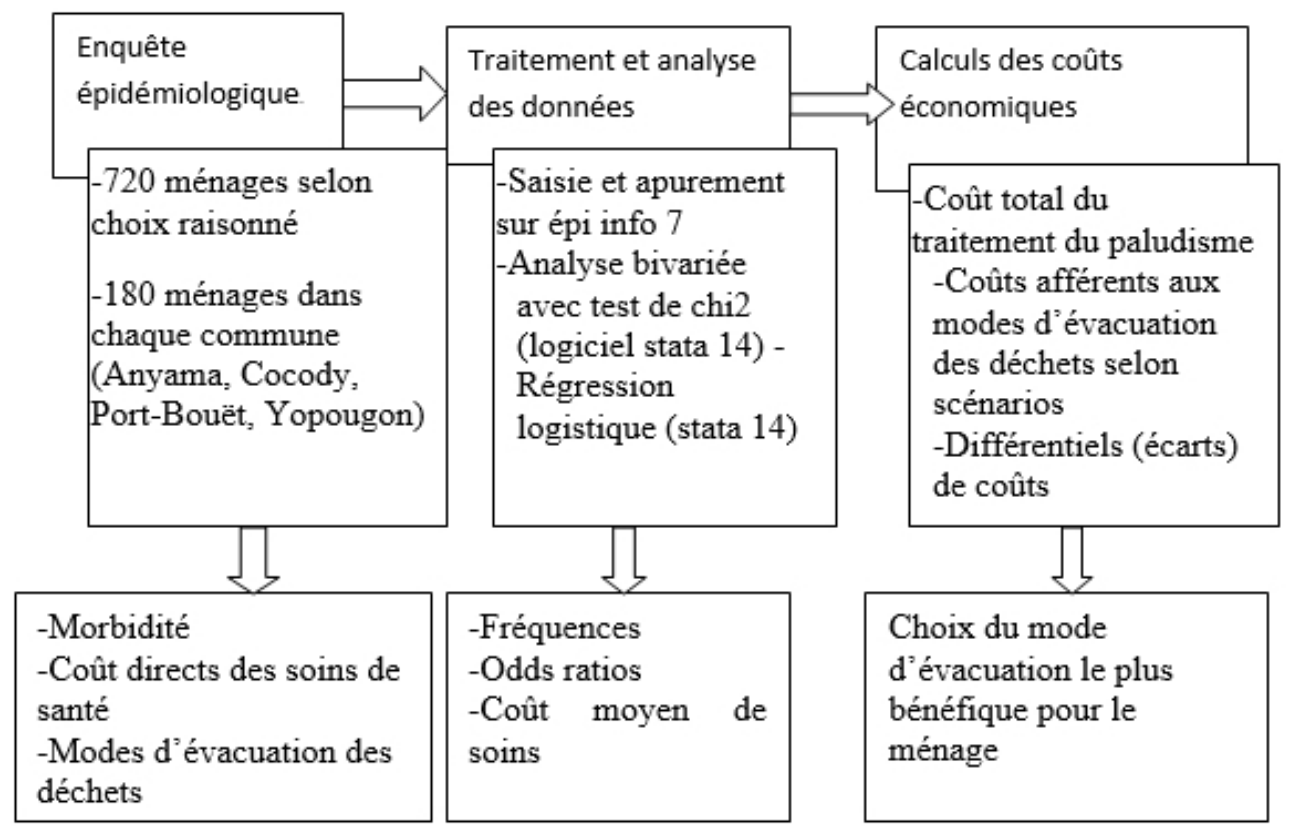

Figure 1. Approche méthodologique générale suivant (Kouadio et al.,2006; GEREP,2009; ANSES,2014; Yvon \& Huchet-Kervella, 2015; Kanj, 2017).

\section{Présentation du modèle}

\section{Variables}

La variable dépendante est la morbidité du paludisme, qui est une variable dichotomique.

Les variables indépendantes sont des variables qualitatives au nombre de deux avec trois modalités chacune. Ces dernières expriment les différentes modes de gestion des déchets solides et liquides par les ménages. Les trois modalités de l'évacuation des ordures solides sont le Coffre, le Pré-collecteur, la Rue, désignant respectivement le fait de déverser les ordures solides, dans un coffre, par l'intermédiaire d'un agent de pré- collecte et dans la rue. Les modalités de l'évacuation des eaux usées sont la fosse septique, la Rue et la Cours. Elles représentent respectivement l'évacuation des eaux usées dans, la fosse septique, la cours et la rue (Koné, 2008).

\section{Modèle}

Le model de régression logistique simple est adapté aux variables qualitative types dichotomiques. IL permet de mieux expliquer les événements tels que, l'avènement ou le non -avènement de la maladie, l'achat ou le non achat d'un produit (Center for Disease Control and Prevention (CDC), 2016) IL permet surtout de faire ressortir les odds ratios qui désignent la chance et / ou le risque d'avènement d'un phénomène étudié. IL est beaucoup 
utilisé en épidémiologie (Bouyer, 2012 ; Center for Disease Control and Prevention (CDC), 2016).

Odd ratio $=\mathrm{P}(\mathrm{Y}=1) / 1-\mathrm{P}(\mathrm{Y}=1)$

Où : $\mathrm{P}(\mathrm{Y}=1)$ égale la probabilité d'apparition de l'évènement étudié.

Et 1-P(Y=1) égale la probabilité de non apparition de l'évènement étudié.

\section{Interprétation du modèle}

Si l'Odd ratio est égale à 2 par exemple, cela signifierait qu'il y a deux fois plus de chance que l'évènement apparaisse sous l'influence de la variable explicative.

Dans cette étude chaque variable explicative comporte 3 modalités. Selon le principe de la régression logistique, le modèle présentera les deux dernières modalités car la première modalité sera prise comme modalité de référence. Les odds ratios seront interprétés comparativement à la modalité de référence (ici, le coffre pour la variable évacuation des ordures solides et la fosse septique pour celle des eaux usées). Dans l'exemple précédent, l'odd ratio égale à 2 signifierait qu'il y a deux fois plus de chance que l'évènement ait lieu sous l'influence de la modalité comparativement à l'influence de la modalité de référence. La modélisation est faite automatiquement sur stata version 14 .

\section{Calcul des coûts afférents aux pratiques de gestion des déchets ménagers}

A partir du coût moyen du traitement de la maladie déclarée par les ménages et le nombre de malades du paludisme de l'échantillon, le coût total du traitement de la maladie est estimé puis les coûts afférents aux différentes pratiques sont déterminées (Kanj, 2017).

$\mathrm{CT}(\mathrm{Palu})=\mathrm{N} \times \mathrm{Cm}(\mathrm{Palu})$ et $\mathrm{Cp}($ Palu $)=$ Odd ratio x P x CT(Palu) (GEREP, 2009). ${ }^{2}$

Avec

CT (Palu) : Coût total du traitement du paludisme

$\mathrm{Cm}$ (Palu) : Coût moyen du traitement du paludisme

$\mathrm{N}$ : Nombre de malades du paludisme

$\mathrm{Cp}$ (Palu): Coût affèrent à la pratique d'évacuation en question

$\mathrm{P}$ : probabilité de la variable d'évacuation de référence

Cette étude met en relief une comparaison entre trois modes d'évacuation, donc entre trois stratégies d'intervention. Ceci permet de simuler des scenarios en vue de la comparaison, comme cela se fait de plus en plus dans les études d'impact (Yvon \& Huchet, 2015).

${ }^{2}$ Selon GEREP (2009), $\mathrm{TCi}=\mathrm{Vi} \mathrm{x} \mathrm{dHi}: \mathrm{TCi}=$ Valeur économique totale de la dégradation sanitaire $\mathrm{i}, \mathrm{Vi}=$ valeur du problème sanitaire $i, \mathrm{dHi}=$ Nombre d'individus affectés par le problème sanitaire i. Dans l'étude-ci, $d H i=N$ x odd ratio $x P$ et Vi=Cm(Palu). La formule utilisée combine la logique de kanj,2017 et la méthode de calcul de GEREP(2009). 


\section{Résultats}

\section{Lien entre le paludisme et les différentes pratiques de gestion}

L'examen du lien existant entre le paludisme et les différents modes d'évacuation des ordures ménagères montre que, la prévalence du paludisme est indépendante du mode d'évacuation des eaux usées mais est significativement corrélée à celle des ordures solides dans les communes d'Anyama, Cocody, Port -Bouet et Yopougon. Par rapport aux eaux usées, la prévalence semble tourner autour de $72 \%$ quel que soit la pratique d'évacuation. Le test de chi2 confirme cette indépendance avec une p-value égale à 0,997 .

Concernant les ordures solides, l'analyse croisée montre que, les ménages qui évacuent leurs ordures, dans un coffre, à travers un agent de précollecte ou dans la rue sont exposés au paludisme à des taux respectifs de 62 $\%, 72 \%$ et $81 \%$. L'évacuation dans la rue présente donc plus de risque de contracter le paludisme tandis que celle faite dans le coffre à ordures est moins risquée pour l'avènement du paludisme. Le p-value du chi2 est égale à 0,005 de cette liaison est égale à 0,005 inférieure à $5 \%$ justifiant la significativité statistique de cette liaison (Brissy et al., 2017) (Tableau 1).

Tableau 1. Lien entre les modes d'évacuation des ordures ménagères et la prévalence du paludisme dans les communes d'Anyama, de Cocody, de Port-Bouet et de Yopougon, Mai-Septembre 2017

\begin{tabular}{llllllll}
\hline Variables & \multicolumn{2}{l}{ Evacuation des eaux usées } & \multicolumn{2}{l}{ Evacuation des ordures solides } & Total \\
\hline P-value du Chi2 & $\mathbf{0 , 9 9 7}$ & & & $\mathbf{0 , 0 0 5}$ & & & \\
\hline Modalités & fosse septiques & Rue & Cours & Coffre & Pré-collecteur & Rue & \\
\hline Palu-Non & $28 \%$ & $28 \%$ & $29 \%$ & $19 \%$ & $28 \%$ & $20 \%$ & $28 \%$ \\
\hline Palu-Oui & $72 \%$ & $72 \%$ & $71 \%$ & $62 \%$ & $72 \%$ & $80 \%$ & $72 \%$ \\
\hline
\end{tabular}

$\mathrm{dHi}=\mathrm{N} \times$ odd ratio $\mathrm{x} \mathrm{P}$ et $\mathrm{Vi}=\mathrm{Cm}(\mathrm{Palu})$. La formule utilisée combine la logique de kanj,2017 et la méthode de calcul de GEREP(2009).

\section{Regression logistique du paludisme et des modes de gestion des ordures ménagères}

La régression logistique simple confirme et précise le lien entre les modes d'évacuations des déchets solides et la maladie du paludisme. Le chi2 avec une p-value égale à 0,005 et inférieur à $5 \%$ montre que le modèle est globalement significatif.

Dans ce modèle la variable de référence est le coffre. Les odds ratio en rapport avec les variables pré-collecteur et Rue sont égale respectivement à 1,61 $(z=2,07 ; p$-value $=0,039<5 \%)$ et $2,53(z=3,18 ; P$-value $=0,001<5 \%)$. Ceci signifierait que les ménages ayant recours à un agent de pré-collecte ont 1,6 fois plus de risque de contracter le paludisme par rapport à ceux qui utiliseraient le coffre. De même, ceux qui déversent leurs ordures directement dans la rue ont 2, 5 fois plus de risque de contracter le paludisme par rapport à ceux qui utiliseraient le coffre. 
La régression atteste donc que le coffre est le mode d'évacuation qui offre le moins de risque de contracter le paludisme comparativement aux deux autres modes d'évacuation des ordures adoptées par les ménages (Yao-Kouassi \& Gohourou, 2018) (Tableau 2).

Tableau 2. Régression logistique entre le paludisme et le mode d'évacuation des ordures solides dans les communes d'Anyama, Cocody, Port- Bouët, Yopougon, Mai- Septembre 2017

\begin{tabular}{llllll}
\hline Paludisme & Odd ratio & Ecart-type & $\mathbf{z}$ & $\mathbf{P}>$ IzI & Intervalle de confiance \\
\hline Pré-collecteur & 1,607089 & 0,3684463 & 2,07 & 0,0039 & $1,025394-2,518773$ \\
\hline Rue & 2,526933 & 0,7357724 & 3,18 & 0,001 & $1,428064-4,471362$ \\
\hline Constante & 1,630435 & 0,305342 & 2,61 & 0,009 & $1,129519-2,353496$
\end{tabular}

Nombre d'observations : 535

Log Likehood : $-312,12692$

Pseudo R2 : 0,0162

LR chi2(2) : 10,58

Prob > Chi2 : 0,005

Coût économique lié au traitement du paludisme selon les modes d'évacuation des ordures ménagères

Le coût moyen déclaré par les populations est de $13883 \mathrm{XOF}^{3}$ par épisode du paludisme. Le nombre d'épisodes de paludisme durant la période d'enquête est de 652 épisodes. Le coût total de traitement du paludisme de la période s'élève à 9051716 XOF et varierait selon que les ménages adoptent un mode d'évacuation des ordures précis. En effet, il est fonction de l'odd ratio et de la proportion des personnes tombées malades du paludisme au sein des ménages qui utilisent le coffre à ordures.

Selon le Tableau II, 0, $62 \%$ des personnes utilisant le coffre à ordures sont tombés malades du paludisme au cours de la période d'enquête. Les odds ratios des variables coffre, pré-collecte et rue sont respectivement de 1, 1, 6 et 2, 5. D'où, trois scénarios se présentent à l'étude selon que les ménages choisissent l'une ou l'autre pratique d'évacuation.

Ainsi, si tous les ménages utilisaient le coffre comme seul moyen d'évacuation des ordures, le coût du traitement de la maladie sur les ménages reviendrait à 5612064 XOF Ce qui diminuerait de $38 \%$ le coût du traitement de la maladie, soit un gain de 3439652 XOF. Cette option représente le premier scénario.

Le second scenario est que tous les ménages optent pour le service d'un agent de pré-collecte. Dans ce cas, le coût du traitement du paludisme diminuerait de $1 \%$, soit un bénéfice de 72414 XOF sur la période d'enquête.

\footnotetext{
${ }^{3}$ XOF est le code de devise du Franc CFA monnaie de huit pays de l'Ouest africain dont fait partie la Côte d'Ivoire) : 13883 XOF=-13 883 F CFA
} 
Le troisième scénario serait que tous les ménages déversent leurs ordures dans la rue. Le coût du traitement augmenterait de $57 \%$, soit une perte de 4978 444 XOF.

Le scénario qui apporte le maximum de bénéfice est celui de l'évacuation des ordures ménagères par le coffre (Tableau 3).

Tableau 3. Coût du traitement du paludisme afférents aux différents modes de gestion et écarts, dans les communes de, Cocody, Anyama, Yopougon et Port-Bouët (Mai -Juillet, 2017)

\begin{tabular}{|c|c|c|c|c|c|}
\hline $\begin{array}{l}\text { Mode } \\
\text { d'évacuation }\end{array}$ & $\mathbf{P}^{*}$ & $\underset{*}{\text { CTM (15 jours) }}$ & $\begin{array}{l}\text { Coût } \\
\text { (XOF) }\end{array}$ & Ecart & $\begin{array}{l}\text { Ecart } \\
(\%)\end{array}$ \\
\hline Coffre & 0,62 & \multirow{3}{*}{9051716} & 5612064 & -3439652 & $-38 \%$ \\
\hline Pré-collecteur & $\begin{array}{l}0,99 \\
2\end{array}$ & & 8979302 & -72414 & $-1 \%$ \\
\hline Rue & 1,55 & & 14030160 & 4978444 & $55 \%$ \\
\hline
\end{tabular}

Une vue générale des pratiques de gestion des ordures solides dans le district d'Abidjan révèle les résultats suivants. La majorité des ménages du district d'Abidjan optent pour une évacuation des ordures solides par un agent pré-collecteur (55\%). $22 \%$ et $23 \%$ d'entre eux évacuent leurs ordures solides respectivement dans la rue et le coffre.

L'analyse selon les communes montre que l'évacuation des ordures solides dans la rue est plus observée à Anyama. En effet $52 \%$ des ménages à Anyama déversent les ordures solides dans la rue. Yopougon semble être la commune qui utilise le moins la rue comme déversoir $(2 \%)$. Elle est celle qui s'attache le plus les services d'un pré-collecteur (92\%) pour évacuer les ordures solides. Les habitants de Cocody viennent en seconde position concernant le déversement des ordures solides dans la rue avec $21 \%$ des ménages reconnaissant y déverser leurs déchets (Figure 3 ; P-value du Chi2=0,000).

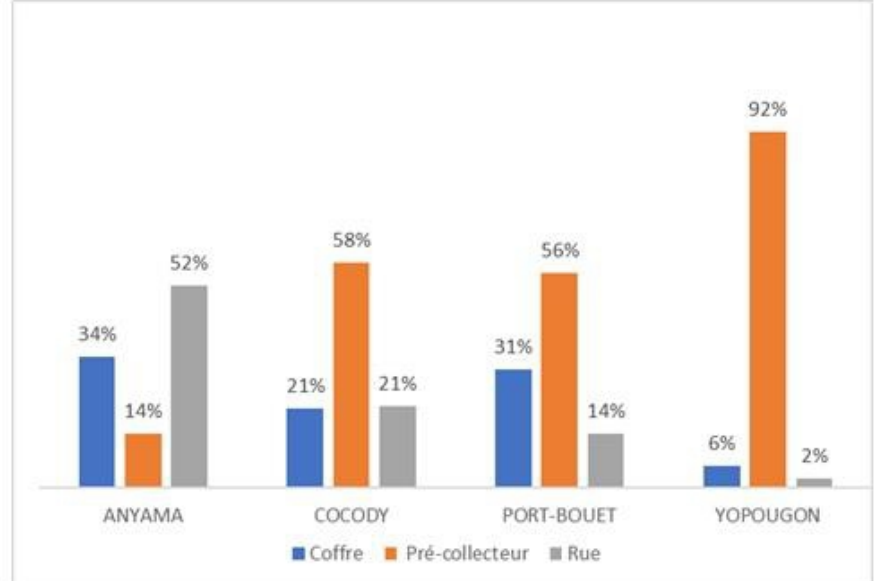

Figure 2. Modes d'évacuation pratiquées par les ménages dans le District d'Abidjan, commune de, Anyama, Cocody, Port-Bouët et Yopougon, Mi-Septembre 201 


\section{Discussion}

La présente étude a eu pour objectif d'estimer l'influence des modes de gestion des ordures ménagères sur le fardeau économique du paludisme dans les communes de, Anyama, Cocody, Yopougon et Port-Bouët.

Pour ce faire il a fallu d'abord estimer le type de lien existant entre les différents modes d'évacuations des ordures ménagères et la prévalence du paludisme. Puis établir éventuellement, la fonction dose- réponse entre le paludisme et les modes d'évacuation des ordures ménagères. Enfin déterminer les coûts économiques directs du paludisme afférents aux modes d'évacuation des ordures ménagères.

Pour estimer le type de lien pouvant exister entre les différentes pratiques d'évacuations des ordures ménagères et la prévalence du paludisme, des tests de chi2 ont été réalisés entre la variable du paludisme et les variables des différents modes de gestion des déchets ménagers (solides et liquides).

Concernant le lien entre l'avènement du paludisme et l'évacuation des eaux usées, l'étude montre qu'il n'existe pas de lien significatif statiquement entre ces deux variables. Quel que soit le mode d'évacuation du ménage la prévalence du paludisme est de $72 \%$, précisément de 72,03\%, 71,88\% et $71,43 \%$ respectivement pour l'évacuation des eaux usées dans la fosse septique , la Rue et la Cours (P-value du Chi2=0,997). Brissy et al. (2017) ont trouvé des résultats similaires dans leur étude sur «Les facteurs de risques écologiques et socioéconomiques associes au paludisme dans les quartiers de la ville de Bouaké». Selon ces auteurs «les morbidités de paludisme à l'échelle des quartiers ne dépendent pas des modes d'évacuation des eaux usées ». Ils ont trouvé une $\mathrm{P}$-value du Chi2 égale à 0,99 entre l'évacuation des eaux usées et le paludisme. C'est seulement dans les quartiers où il existe à la fois une stagnation d'eaux dans lesquels baignent les ordures solides en permanence que le risque de contracter le paludisme est avéré statistiquement (Brissy et al., 2017).

En revanche, le lien entre l'avènement du paludisme et l'évacuation des déchets solides révèle une corrélation significativement positive. Les taux de prévalence du paludisme sont de $61,98 \%, 72,38 \%$ et 80, $47 \%$ respectivement pour l'évacuation, dans le coffre à ordures, par un agent et dans la rue.

Ainsi, une fonction dose-réponse a pu être établie entre l'avènement du paludisme et les pratiques de gestion des déchets solides. Cette dernière qui est une régression logistique, présente des odd ratio de 1,61 pour l'évacuation par un agent de pré-collecte et de 2,53 pour l'évacuation des déchets solides dans la rue. La variable de référence est le coffre. Le chi2 global du modèle a une valeur égale à 0,005 . Celui de la variable de Pré-collecte est égal à 0 , 0039 et celui de la variable Rue a une valeur de 0,001. Selon ces résultats-ci, 
les ménages ayant recours à un agent de pré-collecte pour évacuer leurs ordures ont 1,6 fois plus de risque de contracter le paludisme par rapport à ceux qui utiliseraient le coffre à ordure. De même, ceux qui déversent leurs ordures ménagères dans la rue seraient 2, 5 fois plus exposés au paludisme comparativement à ceux qui ont recours au coffre. Le mode d'évacuation des ordures solides dans le coffre à ordures serait donc le plus indiqué par rapport aux deux autres modes. Avoir pour habitude de jeter les ordures dans la rue entraine une cohabitation avec ces derniers. Or la cohabitation avec les ordures ménagère solides est source potentiels du paludisme (Sy et al., 2011; Somé et al., 2014; Brissy et al., 2017). Par ailleurs, l'enlèvement des ordures ménagères par un agent semble plus commode pour la population abidjanaise et est majoritairement utilisé (Yao-kouassi, 2018). Dans cette étude, c'est environ $55 \%$ des ménages qui a recours aux agents de pré-collecte. Terrabo (2010) a estimé le recours à un agent de pré-collecte à Abidjan à $48 \%$ des ménages tandis que Yao-kouassi (2010) l'a estimé à $75 \%$ pour son étude sur la gestion des déchets ménagers dans les communes de Cocody et de Yopougon (Kouamé, 2015). Seulement, il arrive que le plus souvent ces agents pré-collecteurs se contentent de reverser les ordures dans la rue, au lieu de les envoyer dans les centres de groupages (Eviar et al., 2013 ; Yao -Kouassi \& Gohourou, 2018). C'est pourquoi, ce procédé d'évacuation des déchets prisé par les ménages serait plus exposant à la maladie du paludisme comparativement à l'utilisation du coffre. Ce dernier mode exécuté par le ménage lui-même garantirait l'efficacité de l'évacuation des déchets. Le précollecteur qui se charge d'évacuer les ordures pour les ménages semble beaucoup influencé par le gain. IL privilégierait les ménages les plus offrant au détriment des autres ménages ; se laissant guidé par une «logique pécuniaire » qui accentuerait les inégalités d'accès à la collecte des ordures. (Yao-Kouassi \& Gohourou, 2018, p.1).

De ce qui précède, l'estimation du coût économique issu du choix du mode d'évacuation des ordures ménagères a tenu compte de trois scénarios possibles.

Le premier scénario est celui où tous les ménages utiliseraient le coffre comme seul moyen d'évacuation des ordures. Le coût du traitement du paludisme s'élèverait à 5612064 XOF au lieu de 9025636 XOF sur la période d'enquête. Ceci contribuerait à une diminution du coût de traitement du paludisme de $38 \%$; soit à un bénéfice de 3439652 XOF.

Le second scenario serait que les ménages optent tous pour le service d'un agent de pré-collecte. Dans ce cas, le coût du traitement du paludisme diminuerait de $1 \%$, ce qui représenterait un gain de 72414 XOF.

Le troisième scénario présente des ménages déversant tous leurs ordures dans la rue. Le coût du paludisme augmenterait de $55 \%$ et susciterait une perte de 4978444 XOF. Le mode d'évacuation qui apporte le maximum 
de bénéfice est celui effectué au travers d'un coffre avec un gain de $38 \%$ sur le coût du traitement du paludisme.

\section{Conclusion}

Cette étude a eu pour objectif principal d'appréhender le type de corrélation existant entre le coût du traitement du paludisme et les différentes pratiques de gestion des déchets ménagers dans le district d'Abidjan, spécialement dans les communes de Cocody, Anyama, Port- Bouët et Yopougon. Pour ce faire, d'abord le lien entre les différents modes d'évacuations des ordures ménagères et la prévalence du paludisme a été estimé. Puis, la fonction dose- réponse entre la morbidité du paludisme et les modes d'évacuation des ordures ménagères a été établie. Et enfin, les coûts économiques directs du paludisme afférents aux modes d'évacuation des ordures ménagères ont été déterminés. Il est ressorti conformément aux hypothèses de la recherche que, d'une part le paludisme est positivement corrélé au mode d'évacuation des ordures solides. D'autre part, les ordures ménagères directement évacuées dans le coffre exposent moins au paludisme engendrant ainsi des bénéfices économiques pour le ménage. Il est bon que les autorités locales encouragent les populations à évacuer leurs ordures dans des coffres adaptés. Par ailleurs que les causes profondes de l'attitude peu professionnelle des agents de pré-collecte, majoritairement sollicités par les ménages soient élucidées.

\section{References:}

1. Agence Nationale de Sécurité Sanitaire de l'alimentation,de l'environnement et du travail (ANSES) (2014). Etude exploratoire menée en France, P. Coût socio-économique de la pollution de l'air intérieur.

2. Bézy, O. (2009). La santé c'est la vie dans le silence des organes ». La revue lacanienne, 3(1), 47-50. doi:10.3917/1r1.091.0047

3. Bouyer, J. (2012). Régression logistique - Modélisation des variables quantitatives. Master. Epidémiologie quantitative, Master Recherche Santé Publique. Paris Sud - UVSQ - Paris Descartes - UPEC, ffcel$00794996 f$.

4. Brissy, A.O., Valère Kramo, V.Y., \& Kouassi, K. (2017). Les facteurs de risques écologiques et socio-économiques associés au paludisme dans les quartiers de la ville de Bouaké.

5. Center for Disease Control and Prevention (CDC) (2016). Manuel de formation au logiciel epi info ${ }^{\mathrm{TM}} 7,152 \mathrm{p}$. 
6. Deza, A.D. (2017). Cartographie de la pauvreté non financière dans le district d'Abidjan à partir du recensement général de la population et de l'habitat 2014 de la Côte d'Ivoire.

7. Dongo, K. (2006). Analyse des déficiences dans la gestion du drainage urbain et des déchets solides et liquides dans les quartiers précaires de Yopougon, Abidjan, Côte d' Ivoire: approche cartographie-SIG, modélisation et socio anthropologique. Thèse Unique, Université de Cocody Abidjan, 230 p.

8. Eviar, O.B., Koffi, A., \& Téré, G. (2013). Stratégies de gestion des cadres et conditions de vie des populations à Abobo. European Scientific Journal, ESJ, 9(29).

9. GEREP (2009).

http://www.environnement.gov.tn/fileadmin/medias/pdfs/projet_etud e/1_2.pdf

10. Charron, D.F. (Ed.) (2014). La Recherche Écosanté en pratique: Applications novatrices d'une approche écosystémique de la santé (Vol. 2). Springer.

11. Kanj, O. (2017). Evaluation économique de la prise en charge de l'endométriose (Doctoral dissertation, Université Clermont Auvergne).

12. Koné, B. (2008). Pollution lagunaire, risques sanitaires et environnementaux dans trois villages péri-urbains de la commune de Yopougon (Abidjan, Côte d'Ivoire). Thèse unique de doctorat, Université d'Abobo-Adjamé, Abidjan. 286p.

13. Kouadio, A.S., Cissé, G., Obrist, B., Wyss, K. \& Zingsstag, (2006). Fardeau économique du paludisme sur les ménages démunis des quartiers défavorisés d'Abidjan, Côte d'Ivoire. VertigO-la revue électronique en sciences de l'environnement, (Hors-série 3).

14. Kouamé, PF. (2013). Applicabilité de la redevance incitative d'enlevement des ordures menageres en cote d'ivoire: cas des communes de cocody et de yopougon. journal scientifique européen, 9 (17).

15. Kouamé, P.F. (2015). Approche de financement de la filière de gestion des ordures ménagères en Côte d'Ivoire : cas du district d'Abidjan. Thèse unique de doctorat, Université Nangui-Abrogoua, Abidjan. $194 \mathrm{p}$

16. N'guettia, K.Y. (2010). Gestion des ordures ménagères d'Abidjan : Diagnostic, Mémoire de master spécialisé en génie sanitaire et Environnement, Fondation 2ie, Burkina Faso, 63 p. 23.

17. OMS/RBM (2001). Aspects économiques du paludisme, Roll Back Malaria, Genève 
18. Opio-Odongo, J. (2013). Avenir de l'environnement en Afrique (AEO 3) : notre environnement, notre santé; résumé à l'intention des décideurs, PNUE.

19. Organisation Mondiale de la Santé (OMS) (2007). Prévenir la maladie grâce à un environnement sain, $19 \mathrm{p}$.

20. Organisation Mondiale de la Santé (OMS) (2010). Maladies transmissibles : profil épidémiologique, Côte d'Ivoire, 294 p.

21. Ouellet, E., Belley-Ferris, I., \& Leblond, S. (2005). Guide d'économétrie appliquée pour Stata Pour ECN 3950 et FAS 3900. University of Montreal.

22. Programme des Nations unies pour le Développement (PNUD) (2013). Diagnostic et plans d'amélioration des quartiers précaires des 13 communes du district d'Abidjan, Rapport de synthèse, $31 \mathrm{p}$.

23. Somé, Y. S. C., Soro, T.D., \& Ouedraogo, S. (2014). Étude de la prévalence des maladies liées à l'eau et influences des facteurs environnementaux dans l'arrondissement de Nomgr-Masson: cas du quartier Tanghin (Ouagadougou-Burkina Faso). International Journal of Biological and Chemical Sciences, 8(1): 289-303.

24. Sy, I., Koita, M., Traoré, D., Keita, M., Lo, B., Tanner, M., \& Cissé, G. (2011).Vulnérabilité sanitaire et environnementale dans les quartiers défavorisés de Nouakchott (Mauritanie): analyse des conditions d'émergence et de développement de maladies en milieu urbain sahélien. VertigO-la revue électronique en sciences de l'environnement, 11 (2) : 2-17.

25. Terrabo Ingénieurs Conseil (2010). Étude de caractérisation des déchets urbains du District d'Abidjan 107p.

26. Vaughan, J.P. \& Morrow, R.H. (1991). Manuel d'épidémiologie pour la gestion de la santé au niveau du district. Ed. Jouve, OMS, Genève, $187 \mathrm{p}$.

27. Yao-Kouassi, Q.C. \& Gohourou, F. (2018). L'influence de la précollecte des ordures ménagères sur la qualité de la salubrité dans le District d'Abidjan (Côte-d'Ivoire).

28. Yao-Kouassi, Q.C. (2010). A la recherche d'une synergie pour la gestion des déchets ménagers en Côte d'Ivoire : cas du district d'Abidjan (Doctoral dissertation, Le Mans).

29. Yvon, J.M. \& Huchet, K.C. (2015). Évaluation de l'impact sanitaire de la pollution atmosphérique urbaine dans l'agglomération d'Annecy, 2009-2011. Saint-Maurice: Institut de veille sanitaire. 